\title{
28 Resarch Square \\ The performance of Chest CT and Its Imaging Features for Diagnosing Coronavirus Disease 2019: a Meta-analysis
}

\section{Shuo Zhang}

Peking Union Medical College Hospital, Peking Union Medical College and Chinese Academy of Medical Sciences

Zhewei Zhao

Peking Union Medical College Hospital, Peking Union Medical College and Chinese Academy of Medical Sciences

Chen Li

Peking Union Medical College Hospital, Peking Union Medical College and Chinese Academy of Medical Sciences

Wen Zhang

Peking Union Medical College Hospital, Peking Union Medical College and Chinese Academy of Medical Sciences

Shuyang Zhang ( $\sim$ shuyangzhang103@nrdrs.org )

Peking Union Medical College Hospital, Peking Union Medical College and Chinese Academy of Medical Sciences

\section{Systematic Review}

Keywords: COVID-19, imaging, diagnosis, RT-PCR, meta-analysis

Posted Date: May 15th, 2020

DOI: https://doi.org/10.21203/rs.3.rs-28613/v1

License: (c) (i) This work is licensed under a Creative Commons Attribution 4.0 International License. Read Full License 


\section{Abstract}

Early diagnosis and isolation of cases are particularly crucial for coronavirus disease 2019 (COVID-19) in global pandemic. The aim of this study is to determine the diagnostic performance of chest computed tomography (CT) and imaging features for diagnosing COVID-19. Diagnostic accuracy studies of CT and RT-PCR in patients with clinically suspected COVID-19, which were published up to April 25th, 2020 from MEDLINE, EMBASE, and the Cochrane Library. Twelve studies $(n=2,204)$ were included. The pooled sensitivity, specificity, likelihood ratio (PLR), negative likelihood ratio (NLR), and diagnostic odds ratio (DOR) of chest CT for detecting COVID-19 were 94.5\% (95\% confidence interval (Cl) 89.5 to $97.2 \%$ ) and $41.8 \%$ (95\% Cl 24.2 to $61.6 \%), 1.6$ (95\% Cl: $1.6-2.3), 0.13$ (95\% Cl: 0.06-0.31), and 12.4 (95\% Cl: 4.0-38.5), respectively. Initial RT-PCR revealed a better diagnostic performance. Peripheral lesions, bilateral involvement, multiple lesions, and ground-glass opacities (GGO), revealed to be with better diagnostic value than other CT manifestations. Using chest CT for COVID-19 diagnosis has a high sensitivity and a relatively low specificity. Bilateral multiple peripheral lesions and GGO revealed to be with better diagnostic value. For areas with high prevalence, chest $\mathrm{CT}$ could be a good screening test to preliminary screen patients with COVID-19 quickly.

\section{Introduction}

The coronavirus disease 2019 (COVID-19) is caused by severe acute respiratory syndrome coronavirus 2 (SARS-CoV-2), leading to a global pandemic and public health emergency. Since stringent containment measures have proved to interrupt COVID-19 transmission effectively, early diagnosis and isolation of cases are particularly crucial. To date, the gold standard for the definitive diagnosis of COVID-19 remains to be reverse-transcriptase polymerase chain reaction (RTPCR). However, there are several disadvantages of RT-PCR test, including false-negative results caused by insufficient cellular material or inadequate detection and extraction techniques and shortage of testing kits in some areas ${ }^{1}$. Thus, figuring feasible screening strategies with good diagnostic performance is necessary.

Chest computed tomography (CT) demonstrates typical radiographic features in patients with COVID-19, including bilateral pulmonary parenchymal ground-glass and consolidative pulmonary opacities, sometimes with a rounded morphology and a peripheral lung distribution ${ }^{2}$. In this context, chest CT has been suggested to be an alternative diagnostic tool for COVID-193-5. In order to screen COVID-19 as much as possible in Hubei province, China used to use typical chest CT manifestation combined with clinical symptoms as a clinical diagnostic criteria for COVID-196 ${ }^{6}$ However, the pooled sensitivity of chest CT was only $37 \%(95 \% \mathrm{Cl}: 26-50 \%)^{7}$. Meanwhile, routine CT scan for each patient suspected of COVID-19 would bring high workload and increase infection risk for hospital staffs. Thus, some organizations did not recommend routine CT screening or regarding CT scan as a first-line imaging modality for COVID-198. In this condition, clarification the role of chest CT with high level of evidence is required to guide clinical decision making.

Therefore, we performed the present meta-analysis to determine the diagnostic value of CT compared for diagnosing COVID-19.

\section{Results}

\section{Characteristics and quality of the included studies}

We identified 75 publications in MEDLINE, 71 publications in Embase, 5 publications in the Cochrane Library, and 2 publications manually searched from reference lists. Of these 153 studies, 12 studies $4,9,18,19,10-17(n=2,204)$ met the inclusion criteria and were included (Appendix Figure 1). One study used clinical diagnostic criteria of COVID-19, but the details provide subgroup data in which the patients were diagnosed with RT-PCR test and received chest CT test ${ }^{14}$. The 
data from this subgroup were used in the present meta-analysis. Another study excluded patients with negative CT scan results inappropriately, the original data with all patients that met our selection criteria were used in this meta-analysis. ${ }^{16}$

The characteristics of the nine included studies are summarized in Table 1. The mean age of the participants ranged from 40.0 to 58.2 years old, with 45.5 to $59.8 \%$ males. Nine of the studies were performed in China, and the other two studies were carried out in Italy and Japan, respectively. One case control study recruited COVID-19 patients in China and control group in America. Sample sizes ranged from 21 to 1014 participants.

\section{Quality assessment}

The methodological quality of studies was assessed with QUADAS-2. (Appendix Figure 2). Some study characteristics that might increase the risk of bias were identified. The main concerns of high risk studies focused on patient selection and flow and timing domains. Bai's study was a case-control study that was not a random sample of patients clinically suspected COVID-19 ${ }^{11}$, while Li's study and Miao's study ${ }^{18}$ only focused on patients with abnormal chest CT among the COVID-19 suspected population ${ }^{12}$. In Himoto's study, some of the control group patients were confirmed negative for COVID-19 by careful observation more than 2 weeks without RT-PCR test ${ }^{10}$. Due to the different diagnostic criteria used in Xiong's study ${ }^{16}$, the patient flow could introduce bias with high risk and only part of the valid data were analyzed in our meta-analysis. Most studies did not clarify whether RT-PCR results were interpreted without knowledge of the results of chest CT.

\section{Diagnostic performance of chest CT compared with initial RT-PCR for COVID-19}

Forest plots of the sensitivity and specificity of chest CT and initial RT-PCR assays for diagnosing COVID-19 were shown in Figures 1. The following pooled parameters of chest CT were calculated over eight studies with both sensitivity and specificity data: sensitivity, 94.5\% (95\% Cl: 89.5-97.2\%); specificity, 41.8\% (95\% Cl 24.2 to 61.6\%); PLR, 1.6 (95\% Cl: 1.62.3); NLR, 0.13 (95\% Cl: 0.06-0.31); and DOR, 12.4 (95\% Cl: 4.0-38.5), $\mathrm{I}^{2}=93 \%$. For initial RT-PCR test, pooled sensitivity, 95.1\% (95\% Cl: 87.7-100.0\%); specificity, 100.0\% (95\% Cl: 99.3-100.0\%); PLR, 119.6 (95\% Cl: 22.6-633.5); NLR, 0.13 (95\% Cl: 0.03-0.52); and DOR, 921 (95\% Cl: 74-11496), $\mathrm{l}^{2}=67 \%$. (Table 2) The corresponding symmetric SROC curves were plotted and shown in Figure 2. Initial RT-PCR revealed a better diagnostic performance compared to chest CT.

\section{Estimated posterior probabilities according to COVID-19 prevalence}

The prior probabilities of COVID-19 were estimated from the prevalence data. In a previous meta-analysis, the pooled prevalence of COVID-19 was $38 \%{ }^{7}$. As for close contacts of confirmed COVID-19 patients, secondary attack rate ranged from 0.45 to $5 \%$ among general close contacts and 3 to $10 \%$ among household members. We used prior probabilities of $1 \%, 10 \%$, and $38 \%$ to estimate the Bayesian posterior probabilities. The estimated PPV of chest CT were $2 \%, 15 \%$, and $50 \%$ at a disease prevalence of $1 \%, 10 \%$ and $38 \%$, respectively. While the estimated NPV of chest CT were $0.1 \%, 1 \%$, and $7 \%$ at a disease prevalence of $1 \%, 10 \%$ and $38 \%$, respectively. (Figure 3 )

\section{Comparison of different chest CT manifestations in diagnosing COVID-19}

To further observe the diagnostic performance of different chest CT manifestations, the diagnostic values were summarized in Table 2. Forest plots of CT manifestations were shown in Figure 4 while the corresponding SROC curves were shown in Figure 5. Peripheral lesions, bilateral involvement, multiple lesions, and ground-glass opacities (GGO), revealed to be with better diagnostic value than other $\mathrm{CT}$ manifestations. Meanwhile, pleural effusion, consolidation, and lymphadenopathy were with worst diagnostic performance.

\section{Publication bias in the literature evaluation}


Publication bias was assessed and presented in Appendix Figure 3. The Deeks' test showed a statistically significant value $(p<0.001)$ in initial RT-PCT test, indicating that there was potential publication bias. While no significant publication bias was found in other tests evaluated in this meta-analysis. $(p>0.05)$

\section{Discussion}

In this meta-analysis, diagnostic value of chest CT, initial RT-PCR, and chest CT manifestations were evaluated. We strictly included diagnostic studies of chest CT and RT-PCR with control groups. Using chest CT for COVID-19 diagnosis has a high sensitivity and a relatively low specificity. Compared with initial RT-PCR, it revealed a worse diagnostic performance. Multiple, bilateral and peripheral lesions as well as GGO revealed to be with better diagnostic value than other CT manifestations.

Early detection and diagnosis of COVID-19 are essential for disease control and treatment. For COVID-19, RT-PCR positivity has been widely recognized to be the diagnostic gold standard for its nearly perfect specificity. Our data revealed high diagnostic accuracy of RT-PCR for COVID-19 diagnosis, which indicated its role cannot be taken over by chest CT. Compared to the sensitivities of initial RT-PCR ranging from $37 \%$ to $71 \%$ reported in early studies from China ${ }^{4,5,20}$, our study revealed a higher pooled sensitivity. This value was similar to that of a recent pooled analysis study. ${ }^{7}$ It probably due to the improvement of RT-PCR test kit and technique. However, there are still several drawbacks of RT-PCR in the clinic all over the world: (1) low sensitivity in some regions due to immature development of detection technology, different manufacturers, low patient viral load, or improper clinical sampling5; (2) not available worldwide (especially in developing countries and some area with high prevalence); (3) time consumption for the result. The screening strategies might vary from different areas according to prevalence and the capacity of health systems. But for areas with high prevalence, in order to preliminary screen patients with COVID-19 quickly and isolate them from the public, a screening test with higher sensitivity appears to be warranted.

In this meta-analysis, chest CT was shown to be with high sensitivity for screening COVID-19. As a common tool of examination with good accessibility, increasing reports have focused on the use of CT in the diagnosis of pneumonia ${ }^{21}$. In COVID-19, CT scan could provide information about differential diagnosis, typing, and disease severity. Studies revealed good consistency and reliability in diagnosing COVID-19 among radiologists with different levels of professional experience and nationalities ${ }^{10,11}$. Performing chest CT may be a helpful test for patients suspected of COVID-19. However, with extremely low specificity, the diagnostic accuracy of chest CT was proved to be lower than RT-PCR in this metaanalysis. We also should have a clear recognition of the limitations of CT imaging in patient with COVID-19. The differences of prior probabilities might affect the results in differential diagnosis by chest CT, caused by factors including the region COVID-19 prevalence ${ }^{10}$. Meanwhile, widespread CT screening would definitely increases burden on medical expenses and pose significant risk for hospital staffs ${ }^{22}$.

Another key finding from our analysis is that we revealed the diagnostic value of typical chest CT scan imaging features. Multiple, bilateral and peripheral lesions and GGO were the top three features with better diagnostic performance. While pleural effusion, lymphadenopathy and consolidation were with worst diagnostic value. This was consistent with previous studies and clinical experiences. Majidi et $\mathrm{al}^{22}$ did pooled analysis of chest CT manifestation with occurrence rate as follows: GGO $74 \%$, bilateral distribution $76 \%$, peripheral distribution $66 \%$, consolidation $36 \%$, pleural effusion $7 \%$, crazy paving appearance $21 \%$, lymphadenopathy $5 \%$. However, the imaging findings reported for COVID-19 were similar to other virus pneumonia, including severe acute respiratory syndrome (SARS)-CoV and Middle East respiratory syndrome (MERS)-CoV23. It is crucial to know the diagnostic value of each typical CT feature beyond the simple occurrence rates. Surprisingly, although nearly one third patients presented with consolidation in chest CT, the diagnostic value was pretty low. Due to the limited number of studies enrolled with high heterogeneity, our results were erratic. Further studies are still needed for better understanding of the CT scan features in diagnosis. 
However, there were high heterogeneities in our included studies and the sources of heterogeneity were explored. Except for the differences between study designs and source of participants as mentioned before, the unclear chest CT diagnostic criteria might comprise another source of such heterogeneity. Although there was a consensus of typical chest CT manifestation in patients with COVID-19, there was a lack of clear declaration of the detailed diagnostic criteria in most of included studies. Meanwhile, the chest CT was reviewed by radiologists with different experiences.

These findings have practical implications. It is a complex issue to decide what kind of chest CT role would play in patients with COVID-19, a supplemental role or an alternative role for RT-PCR. With the help of diagnostic performance data of chest CT and initial RT-PCR with high level of evidence, the clinicians and policy makers might achieve the right balance of COVID-19 management. RT-PCR could not be replaced in diagnosis, but how to maximize the value of chest CT needs knowledge and wisdom. The screening and management of COVID-19 should combine the situations of local COVID-19 prevalence, capacity of health systems, and availability of RT-PCR into consideration.

Our study findings must be interpreted considering several limitations. First, the number of diagnostic performance studies was limited. We only included 12 studies in this meta-analysis, although we have tried to include incorporate information from all relevant studies. Thus, one case-control study and several retrospective studies were included. Secondly, the heterogeneity of these studies was also considerable. The major concern was the study population, which varied in several respects. Some studies focused on subgroups from patients suspected of COVID-19, such as virus pneumonia. The unclear definition of CT diagnosis criteria also contributed to the heterogeneity. Meanwhile, the studies were conducted in areas of different prevalence rates. A further limitation was that the limited study number would affect the statistical results. Meanwhile, further subgroup analysis and sensitivity analysis were infeasible due to the rather small number of included studies.

Using chest CT for COVID-19 diagnosis has a high sensitivity and a relatively low specificity. While initial RT-PCR revealed a better diagnostic performance than chest CT. Peripheral lesions, bilateral involvement, multiple lesions, and GGO revealed to be with better diagnostic value than other CT manifestations.

\section{Methods}

\section{Search strategy and selection criteria}

This systematic review and meta-analysis was done according to PRISMA guidelines. We performed a systematic review of articles published up to April 25th, 2020 in Medline, EMBASE, and the Cochrane Library. Keywords included "COVID-19" OR "severe acute respiratory syndrome coronavirus 2" OR "severe acute respiratory syndrome coronavirus 2" OR "2019nCoV" OR "SARS-CoV-2" AND "computed tomography" AND "Reverse Transcriptase Polymerase Chain Reaction" (Appendix Table 1). There was no restriction on publication language. The reference lists of primary studies and previous reviews were also manually searched for additional relevant articles. This study is registered with PROSPERO, number CRD42020181869.

\section{Study selection}

Studies meeting the following inclusion criteria were included: (I) patients: patients clinically suspected COVID-19; (II) diagnostic tool: chest CT; (III) gold reference: positivity of any one of RT-PCR tests; and (IV) outcomes: true positive (TP), false positive (FP), true negative (TN) and false negative (FN) results, which could be extracted or accurately calculated through $2 \times 2$ contingency tables.

The exclusion criteria were as follows: (1) literatures not pertinent to Chest CT and COVID-19 diagnosed without RT-PCR; (2) no control groups; (3) case reports, correspondences, reviews, or any other types of articles without original data. 


\section{Data Extraction}

Two investigators independently extracted data with a predefined data collection form. For each study, first author's surname, publication year, patients' mean age, sex, country, study design, sample size, diagnostic tool, gold standard, TP, FP, TN, and FN were collected. The definition of initial RT-PCR was the first RT-PCR performed in COVID-19 suspected population. Disagreements were determined a third author.

\section{Quality assessment}

The Quality Assessment of Diagnostic Accuracy Studies checklist-2 (QUADAS-2) was used to assess the methodological quality and risks of bias of the studies. Two authors independently abstracted data and performed quality assessment. Disagreements were determined a third author.

\section{Statistical analysis}

Standard methods recommended for diagnostic accuracy meta-analysis were used. A random-effects meta-analysis was carried out to take into account inter-study variability. We used hierarchical summary receiver operating characteristic (HSROC) model and DerSimonian-Lard model (when HSROC was not available due to limited study number) to conduct the meta-analysis. The following indexes of test accuracy together with $95 \%$ confidence intervals ( $95 \% \mathrm{Cls}$ ) of each study were computed: sensitivity, specificity, positive likelihood ratio (PLR), negative likelihood ratio (NLR) diagnostic odds ratio (DOR), area under the receiver operating curve (AUROC), and symmetric summary receiver operating characteristic (symmetric SROC). The degree of heterogeneity was quantified using $\mathrm{I}^{2}$, with $<25 \%, 25 \%-50 \%$, and $>50 \%$ indicating mild, moderate, and high heterogeneity, respectively. Publication bias was assessed using funnel plots. All analyses were performed using Stata (version 14; Stata Corporation, College Station, TX, USA), Meta-DiSc (version 1.4, Hospital Ramony Cajal, Madrid, Spain) and RevMan (version 5.3; The Cochrane Collaboration- Oxford, UK). All statistical tests were twosided, and $p<0.05$ was regarded as statistically significant.

\section{Declarations}

Acknowledgement: The authors apologize to all colleagues whose works have not been separately cited or discussed here due to space or knowledge limitations. This work was supported by the CAMS Innovation Fund for Medical Sciences [No. 2017-I2M-3-001] and the National Key Research and Development Program of China [No. 2016YFC0901500].

Competing interests: No conflict of interests to declare.

Contributions: Shuo Zhang, Zhewei Zhao, and Chen Li collected the data and did the analysis, under the supervision of Shuyang Zhang and Wen Zhang. All authors revised and reviewed the manuscript.

Data availability: The datasets used and/or analyzed during the current study are available from the corresponding author on reasonable request.

\section{References}

1. Lippi, G., Simundic, A.-M. \& Plebani, M. Potential preanalytical and analytical vulnerabilities in the laboratory diagnosis of coronavirus disease 2019 (COVID-19). Clin. Chem. Lab. Med. (2020). doi:10.1515/cclm-2020-0285

2. Chung, M. et al. CT Imaging Features of 2019 Novel Coronavirus (2019-nCoV). Radiology 295, 202-207 (2020).

3. Nair, A. et al. A British Society of Thoracic Imaging statement: considerations in designing local imaging diagnostic algorithms for the COVID-19 pandemic. Clin. Radiol. 75, 329-334 (2020). 
4. Ai, T. et al. Correlation of Chest CT and RT-PCR Testing in Coronavirus Disease 2019 (COVID-19) in China: A Report of 1014 Cases. Radiology 200642 (2020). doi:10.1148/radiol.2020200642

5. Fang, Y. et al. Sensitivity of Chest CT for COVID-19: Comparison to RT-PCR. Radiology 200432 (2020). doi:10.1148/radiol.2020200432

6. The National Health Commission of China. Notice on the issuance of a program for the diagnosis and treatment of novel coronavirus (2019-nCoV) infected pneumonia (fifth edition) (2020/02/21). Available at: http://www.chinacdc.cn/jkzt/crb/zl/szkb_11803/jszl_11815/202002/t20200223_213615.html.

7. Kim, H., Hong, H. \& Yoon, S. H. Diagnostic Performance of CT and Reverse Transcriptase-Polymerase Chain Reaction for Coronavirus Disease 2019: A Meta-Analysis. Radiology 201343 (2020). doi:10.1148/radiol.2020201343

8. The Society of Thoracic Radiology and American Society of Emergency Radiology. STR / ASER COVID-19 Position Statement. (2020/03/11).

9. D., Z. et al. A comparative study on the clinical features of COVID-19 pneumonia to other pneumonias. Clin. Infect. Dis. (2020). doi:10.1093/cid/ciaa247

10. Himoto, Y. et al. Diagnostic performance of chest CT to differentiate COVID-19 pneumonia in non-high-epidemic area in Japan. Jpn. J. Radiol. (2020). doi:10.1007/s11604-020-00958-w

11. Bai, H. X. et al. Performance of radiologists in differentiating COVID-19 from viral pneumonia on chest CT. Radiology 200823 (2020). doi:10.1148/radiol.2020200823

12. D., L. et al. False-negative results of real-time reverse-transcriptase polymerase chain reaction for severe acute respiratory syndrome coronavirus 2: Role of deep-learning-based ct diagnosis and insights from two cases. Korean J. Radiol. 21, 505-508 (2020).

13. C., L. et al. Diagnosis of the Coronavirus disease (COVID-19): rRT-PCR or CT? Eur. J. Radiol. 126, (2020).

14. Xiong, Z. et al. [Construction and evaluation of a novel diagnosis process for 2019-Corona Virus Disease]. Zhonghua Yi Xue Za Zhi 100, E019 (2020).

15. Caruso, D. et al. Chest CT Features of COVID-19 in Rome, Italy. Radiology 201237 (2020). doi:10.1148/radiol.2020201237

16. Cheng, Z. et al. Clinical Features and Chest CT Manifestations of Coronavirus Disease 2019 (COVID-19) in a SingleCenter Study in Shanghai, China. AJR. Am. J. Roentgenol. 1-6 (2020). doi:10.2214/AJR.20.22959

17. He, J.-L. et al. Diagnostic performance between CT and initial real-time RT-PCR for clinically suspected 2019 coronavirus disease (COVID-19) patients outside Wuhan, China. Respir. Med. 105980 (2020). doi:https://doi.org/10.1016/j.rmed.2020.105980

18. Miao, C. et al. Early chest computed tomography to diagnose COVID-19 from suspected patients: A multicenter retrospective study. Am. J. Emerg. Med. (2020). doi:10.1016/j.ajem.2020.04.051

19. Zhu, W. et al. Initial clinical features of suspected coronavirus disease 2019 in two emergency departments outside of Hubei, China. J. Med. Virol. n/a, (2020).

20. Li, Y. et al. Stability issues of RT-PCR testing of SARS-CoV-2 for hospitalized patients clinically diagnosed with COVID19. J. Med. Virol. (2020). doi:10.1002/jmv.25786

21. Claessens, Y.-E. et al. Early Chest Computed Tomography Scan to Assist Diagnosis and Guide Treatment Decision for Suspected Community-acquired Pneumonia. Am. J. Respir. Crit. Care Med. 192, 974-982 (2015).

22. Majidi, H. \& Niksolat, F. Chest CT in patients suspected of COVID-19 infection: A reliable alternative for RT-PCR. Am. J. Emerg. Med. (2020). doi:https://doi.org/10.1016/j.ajem.2020.04.016

23. Kanne, J. P. Chest CT Findings in 2019 Novel Coronavirus (2019-nCoV) Infections from Wuhan, China: Key Points for the Radiologist. Radiology 295, 16-17 (2020). 


\section{Tables}

Table 1 The characteristics of the included studies.

\begin{tabular}{|c|c|c|c|c|c|c|}
\hline Study/Year & Sample Size & Mean Age (years) & Male (\%) & Country & Event (COVID-19/Control) & Design \\
\hline Ai 2020 & 1014 & 51.0 & 46.1 & China & $601 / 413$ & Retrospective \\
\hline Bai 2020 & 424 & 54.4 & 52.4 & China, America & $219 / 205$ & Case control \\
\hline Caruso 2020 & 158 & 57.0 & 52.5 & Italy & $62 / 96$ & Prospective \\
\hline Cheng 2020 & 38 & 45.8 & 45.5 & China & $11 / 27$ & Retrospective \\
\hline He 2020 & 82 & 43.2 & 59.8 & China & $34 / 48$ & Retrospectiv \\
\hline Himoto 2020 & 21 & 55.8 & 50.0 & Japan & $6 / 15$ & Retrospective \\
\hline Li 2020 & 53 & 58.2 & 54.7 & China & $51 / 2$ & Retrospective \\
\hline Long 2020 & 87 & 46.1 & 52.9 & China & $36 / 51$ & Retrospective \\
\hline Miao 2020 & 130 & 43.2 & 59.2 & China & $54 / 76$ & Retrospective \\
\hline Xiong 2020 & 47 & unclear & unclear & China & $20 / 27$ & Unclear \\
\hline Zhao 2020 & 34 & 40.7 & 50.0 & China & $19 / 15$ & Unclear \\
\hline Zhu 2020 & 116 & 40.0 & 48.2 & China & $32 / 84$ & Retrospective \\
\hline
\end{tabular}

Table 2 Pooled diagnostic performance value of chest CT, initial RT-PCR, and chest CT imaging feature. 


\begin{tabular}{|c|c|c|c|c|c|c|c|c|c|c|}
\hline CT manifestation & Study & $\begin{array}{l}\text { COVID- } \\
19\end{array}$ & Control & $\begin{array}{l}\text { Sensitivity } \\
\text { (value [95\% } \\
\text { CI]) }\end{array}$ & $\begin{array}{l}\text { Specificity } \\
\text { (value [95\% } \\
\text { CI]) }\end{array}$ & $\begin{array}{l}\text { PLR } \\
\text { (value [95\% } \\
\text { CI]) }\end{array}$ & $\begin{array}{l}\text { NLR } \\
\text { (value } \\
{[95 \% \mathrm{CI}] \text { ) }}\end{array}$ & $\begin{array}{l}\text { DOR } \\
\text { (value } \\
{[95 \%} \\
\text { CI]) }\end{array}$ & $\begin{array}{l}\text { AUROC } \\
\text { (value } \\
{[95 \%} \\
\text { CI]) } \\
\end{array}$ & $\begin{array}{l}\mathrm{I}^{2} \\
(\%)\end{array}$ \\
\hline Chest CT & 8 & 1060 & 908 & $\begin{array}{l}0.945[0.895, \\
0.972]\end{array}$ & $\begin{array}{l}0.418[0.242, \\
0.616]\end{array}$ & $\begin{array}{l}1.62 \\
{[1.15,2.29]}\end{array}$ & $\begin{array}{l}0.13[0.06, \\
0.31]\end{array}$ & $\begin{array}{l}12.4 \\
{[4.0} \\
38.5]\end{array}$ & $\begin{array}{l}0.90 \\
{[0.87} \\
0.92]\end{array}$ & 93 \\
\hline Initial RT-PCR* & 4 & 657 & 573 & $\begin{array}{l}0.951[0.877, \\
1.000]\end{array}$ & $\begin{array}{l}1.000[0.993, \\
1.000]\end{array}$ & $\begin{array}{l}119.56 \\
{[22.57} \\
633.48]\end{array}$ & $\begin{array}{l}0.13[0.03, \\
0.52]\end{array}$ & $\begin{array}{l}921.4 \\
{[73.8,} \\
11496.1]\end{array}$ & $\begin{array}{l}1.00 \\
{[1.00,} \\
1.00]\end{array}$ & 67 \\
\hline $\begin{array}{l}\text { Air bronchogram } \\
\text { sign }\end{array}$ & 4 & 335 & 310 & $\begin{array}{l}0.412[0.173, \\
0.701]\end{array}$ & $\begin{array}{l}0.821[0.671, \\
0.912]\end{array}$ & $\begin{array}{l}2.31 \\
{[0.95,5.63]}\end{array}$ & $\begin{array}{l}0.71[0.44, \\
1.17]\end{array}$ & $\begin{array}{l}3.2[0.8, \\
12.3]\end{array}$ & $\begin{array}{l}0.76 \\
{[0.72,} \\
0.80]\end{array}$ & 78 \\
\hline $\begin{array}{l}\text { Bilateral } \\
\text { involvement }\end{array}$ & 5 & 330 & 395 & $\begin{array}{l}0.789[0.722, \\
0.844]\end{array}$ & $\begin{array}{l}0.507[0.363, \\
0.650]\end{array}$ & $\begin{array}{l}1.60[1.15, \\
2.23]\end{array}$ & $\begin{array}{l}0.42[0.26, \\
0.66]\end{array}$ & $\begin{array}{l}3.8[1.8, \\
8.4]\end{array}$ & $\begin{array}{l}0.78 \\
{[0.74,} \\
0.82]\end{array}$ & 65 \\
\hline Consolidation & 8 & 1010 & 873 & $\begin{array}{l}0.203[0.089, \\
0.400]\end{array}$ & $\begin{array}{l}0.609[0.387, \\
0.793]\end{array}$ & $\begin{array}{l}0.52[0.32, \\
0.85]\end{array}$ & $\begin{array}{l}1.31[1.03, \\
1.66]\end{array}$ & $\begin{array}{l}0.39 \\
{[0.21} \\
0.75]\end{array}$ & $\begin{array}{l}0.36 \\
{[0.31} \\
0.40]\end{array}$ & 99 \\
\hline $\begin{array}{l}\text { Crazy paving } \\
\text { pattern }\end{array}$ & 5 & 362 & 382 & $\begin{array}{l}0.105 \\
{[0.037,0.262]}\end{array}$ & $\begin{array}{l}0.961 \\
{[0.875,0.989]}\end{array}$ & $\begin{array}{l}2.68 \\
{[0.59,12.23]}\end{array}$ & $\begin{array}{l}0.93 \\
{[0.82,1.05]}\end{array}$ & $\begin{array}{l}2.9[0.6 \\
14.6]\end{array}$ & $\begin{array}{l}0.68 \\
{[0.63} \\
0.72]\end{array}$ & 42 \\
\hline GGO & 9 & 1029 & 936 & $\begin{array}{l}0.657[0.407, \\
0.842]\end{array}$ & $\begin{array}{l}0.593[0.375 \\
0.780]\end{array}$ & $\begin{array}{l}1.61[0.886, \\
2.94]\end{array}$ & $\begin{array}{l}0.58[0.28, \\
1.20]\end{array}$ & $\begin{array}{l}2.8[0.8 \\
10.0]\end{array}$ & $\begin{array}{l}0.66 \\
{[0.62,} \\
0.70]\end{array}$ & 99 \\
\hline $\begin{array}{l}\text { GGO with } \\
\text { consolidation }\end{array}$ & 5 & 364 & 349 & $\begin{array}{l}0.475[0.320, \\
0.634]\end{array}$ & $\begin{array}{l}0.630[0.433, \\
0.792]\end{array}$ & $\begin{array}{l}1.28[0.97 \\
1.69]\end{array}$ & $\begin{array}{l}0.83[0.71, \\
0.98]\end{array}$ & $\begin{array}{l}1.5[1.0, \\
2.3]\end{array}$ & $\begin{array}{l}0.56 \\
{[0.52,} \\
0.60]\end{array}$ & 97 \\
\hline Lymphadenopathy & 7 & 45 & 824 & $\begin{array}{l}0.031[0.016, \\
0.061]\end{array}$ & $\begin{array}{l}0.942[0.876, \\
0.974]\end{array}$ & $\begin{array}{l}0.54[0.16, \\
1.85]\end{array}$ & $\begin{array}{l}1.03[0.97, \\
1.09]\end{array}$ & $\begin{array}{l}0.5[0.1, \\
1.9]\end{array}$ & $\begin{array}{l}0.40 \\
{[0.31} \\
0.48]\end{array}$ & 51 \\
\hline Multiple lesion* & 5 & 642 & 362 & $\begin{array}{l}0.680[0.627, \\
0.729]\end{array}$ & $\begin{array}{l}0.373[0.323- \\
0.425]\end{array}$ & $\begin{array}{l}1.31[0.97 \\
1.75]\end{array}$ & $\begin{array}{l}0.49[0.21- \\
1.16]\end{array}$ & $\begin{array}{l}3.3[0.9 \\
12.7]\end{array}$ & $\begin{array}{l}0.83 \\
{[0.57} \\
1.00]\end{array}$ & 0 \\
\hline Nodule & 4 & 172 & 456 & $\begin{array}{l}0.235[0.157, \\
0.335]\end{array}$ & $\begin{array}{l}0.743[0.666, \\
0.806]\end{array}$ & $\begin{array}{l}0.91[0.63, \\
1.31]\end{array}$ & $\begin{array}{l}1.03[0.92, \\
1.16]\end{array}$ & $\begin{array}{l}0.9[0.5, \\
1.4]\end{array}$ & $\begin{array}{l}0.55 \\
{[0.34,} \\
0.75]\end{array}$ & 25 \\
\hline Peripheral lesion & 5 & 326 & 373 & $\begin{array}{l}0.761[0.666, \\
0.835]\end{array}$ & $\begin{array}{l}0.538[0.387, \\
0.682]\end{array}$ & $\begin{array}{l}1.64 \\
{[1.28,2.12]}\end{array}$ & $\begin{array}{l}0.45[0.36 \\
0.56]\end{array}$ & $\begin{array}{l}3.7[2.5, \\
5.4]\end{array}$ & $\begin{array}{l}0.73 \\
{[0.69} \\
0.77]\end{array}$ & 90] \\
\hline Pleural effusion & 7 & 117 & 752 & $\begin{array}{l}0.024[0.011, \\
0.055]\end{array}$ & $\begin{array}{l}0.856[0.736, \\
0.927]\end{array}$ & $\begin{array}{l}0.17 \\
{[0.58,0.388]}\end{array}$ & $\begin{array}{l}1.14[1.03, \\
1.27]\end{array}$ & $\begin{array}{l}0.2[0.1, \\
0.4]\end{array}$ & $\begin{array}{l}0.04 \\
{[0,} \\
0.11]\end{array}$ & 23 \\
\hline $\begin{array}{l}\text { Reverse Halo } \\
\text { sign* }\end{array}$ & 3 & 14 & 484 & $\begin{array}{l}0.048[0.026, \\
0.081]\end{array}$ & $\begin{array}{l}0.995[0.973, \\
1.000]\end{array}$ & $\begin{array}{l}1.94[0.05 \\
79.72]\end{array}$ & $\begin{array}{l}0.96[0.93, \\
0.99]\end{array}$ & $\begin{array}{l}2.0[0.0 \\
88.7]\end{array}$ & $\begin{array}{l}0.84 \\
{[0.80,} \\
0.87]\end{array}$ & 100 \\
\hline
\end{tabular}

CT, computed tomography; RT-PCR, reverse-transcriptase polymerase chain reaction; PLR, positive likelihood ratio; NLR, negative likelihood ratio; DOR, diagnostic odds ratio; AUROC, area under the receiver operating curve; GGO, ground-glass opacities. *DerSimonian-Laird Model.

\section{Supplementary Material}

Appendix Fig. 1. Flow chart of literature search and study selection

Appendix Fig. 2. Summary of risk of bias and applicability concerns.

Appendix Fig. 3 Publication bias

Appendix Table 1 The detailed research strategy

Figures 
chest CT

$\begin{array}{lrrrrrr}\text { Study } & \text { TP } & \text { FP } & \text { FN } & \text { TN } & \text { Sensitivity }(95 \% \text { CI) } & \text { Specificity }(95 \% \text { Cl) } \\ \text { Ai 2020 } & 580 & 380 & 21 & 105 & 0.97[0.95,0.98] & 0.22[0.18,0.26] \\ \text { Bai 2020 } & 175 & 156 & 44 & 49 & 0.80[0.74,0.85] & 0.24[0.18,0.30] \\ \text { Caruso 2020 } & 60 & 42 & 2 & 54 & 0.97[0.89,1.00] & 0.56[0.46,0.66] \\ \text { Cheng 2020 } & 11 & 22 & 0 & 5 & 1.00[0.72,1.00] & 0.19[0.06,0.38] \\ \text { He 2020 } & 26 & 8 & 2 & 46 & 0.93[0.76,0.99] & 0.85[0.73,0.93] \\ \text { Li 2020 } & 49 & 2 & 2 & 0 & 0.96[0.87,1.00] & 0.00[0.00,0.84] \\ \text { Long 2020 } & 35 & 0 & 1 & 0 & 0.97[0.85,1.00] & \text { Not estimable } \\ \text { Xiong 2020 } & 19 & 8 & 1 & 19 & 0.95[0.75,1.00] & 0.70[0.50,0.86] \\ \text { Zhu 2020 } & 30 & 56 & 2 & 28 & 0.94[0.79,0.99] & 0.33[0.23,0.44]\end{array}$

Sensitivity $(95 \% \mathrm{Cl}) \quad$ Specificity $(95 \% \mathrm{Cl})$

initial RT-PCR

$\begin{array}{lrrrrrr}\text { Study } & \text { TP } & \text { FP } & \text { FN } & \text { TN } & \text { Sensitivity }(95 \% \text { Cl) } & \text { Specificity }(95 \% \text { Cl) } \\ \text { Ai 2020 } & 586 & 0 & 15 & 413 & 0.98[0.96,0.99] & 1.00[0.99,1.00] \\ \text { He 2020 } & 27 & 0 & 7 & 48 & 0.79[0.62,0.91] & 1.00[0.93,1.00] \\ \text { Long 2020 } & 30 & 0 & 6 & 51 & 0.83[0.67,0.94] & 1.00[0.93,1.00] \\ \text { Xiong 2020 } & 14 & 0 & 6 & 27 & 0.70[0.46,0.88] & 1.00[0.87,1.00]\end{array}$

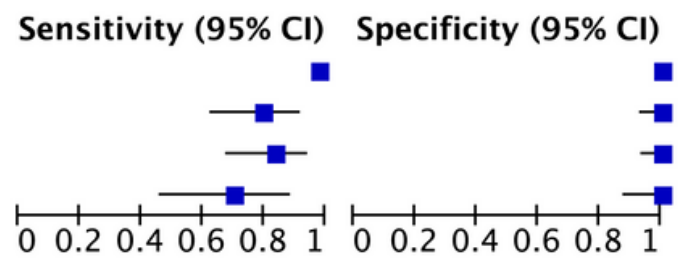

Figure 1

Forrest plot illustrating the diagnostic performance of chest CT and RT-PCR for COVID-19 


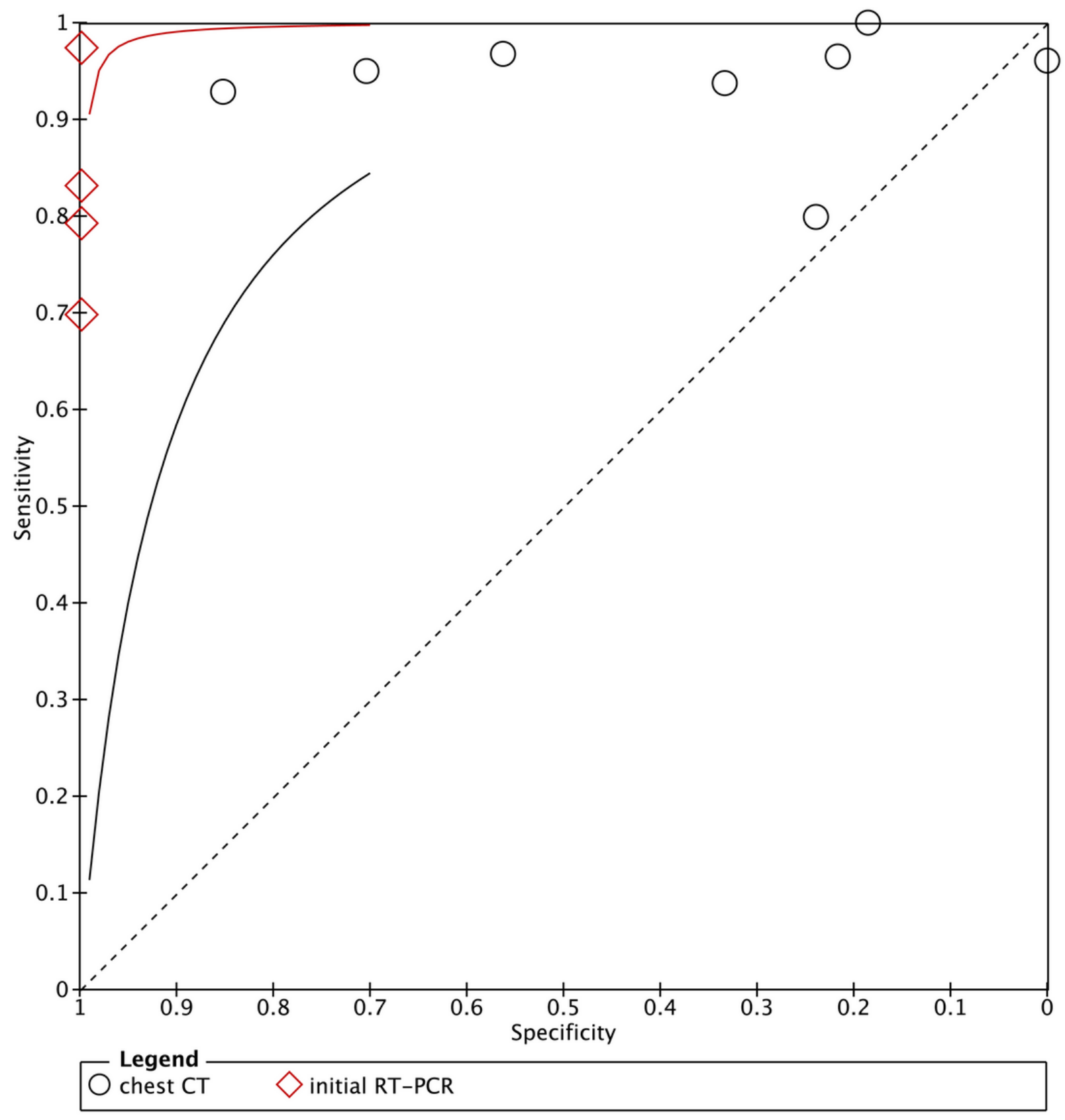

Figure 2

Symmetric summary receiver operating characteristic (symmetric SROC) of chest CT and RT-PCR for diagnosing COVID19 

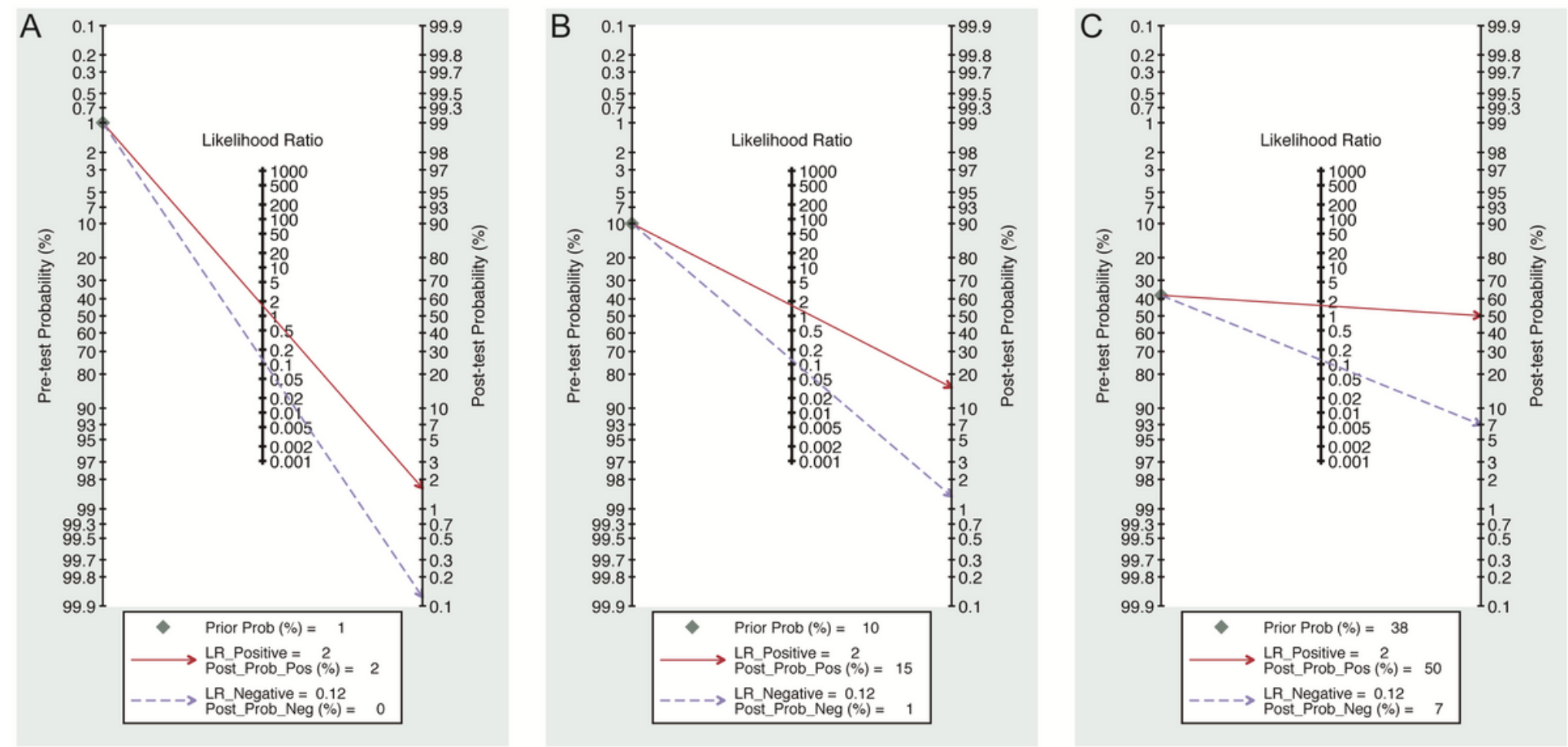

Figure 3

Bayesian posterior probabilities estimated according to COVID-19 prevalence Prior probabilities of $0.01(A), 0.10(B)$, and $0.38(\mathrm{C})$. 


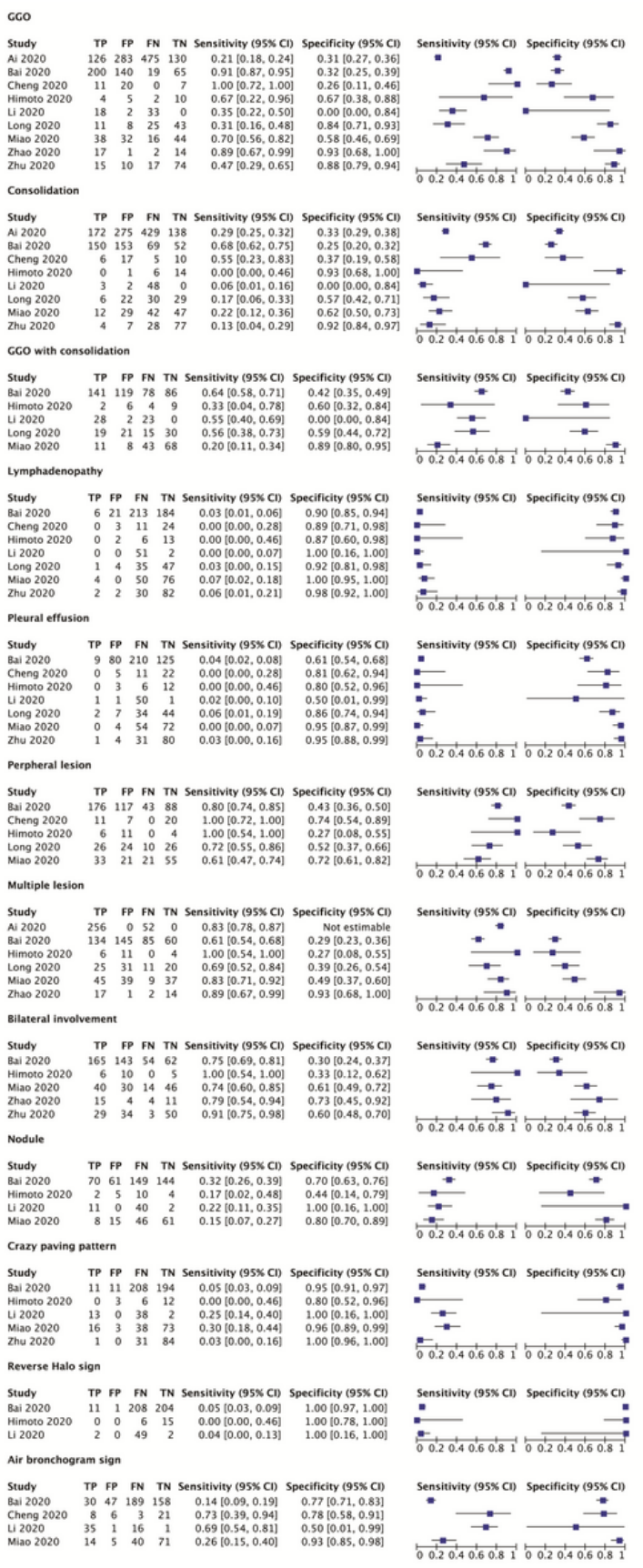

\section{Figure 4}

Forrest plot illustrating the diagnostic performance of chest CT imaging features for COVID-19 


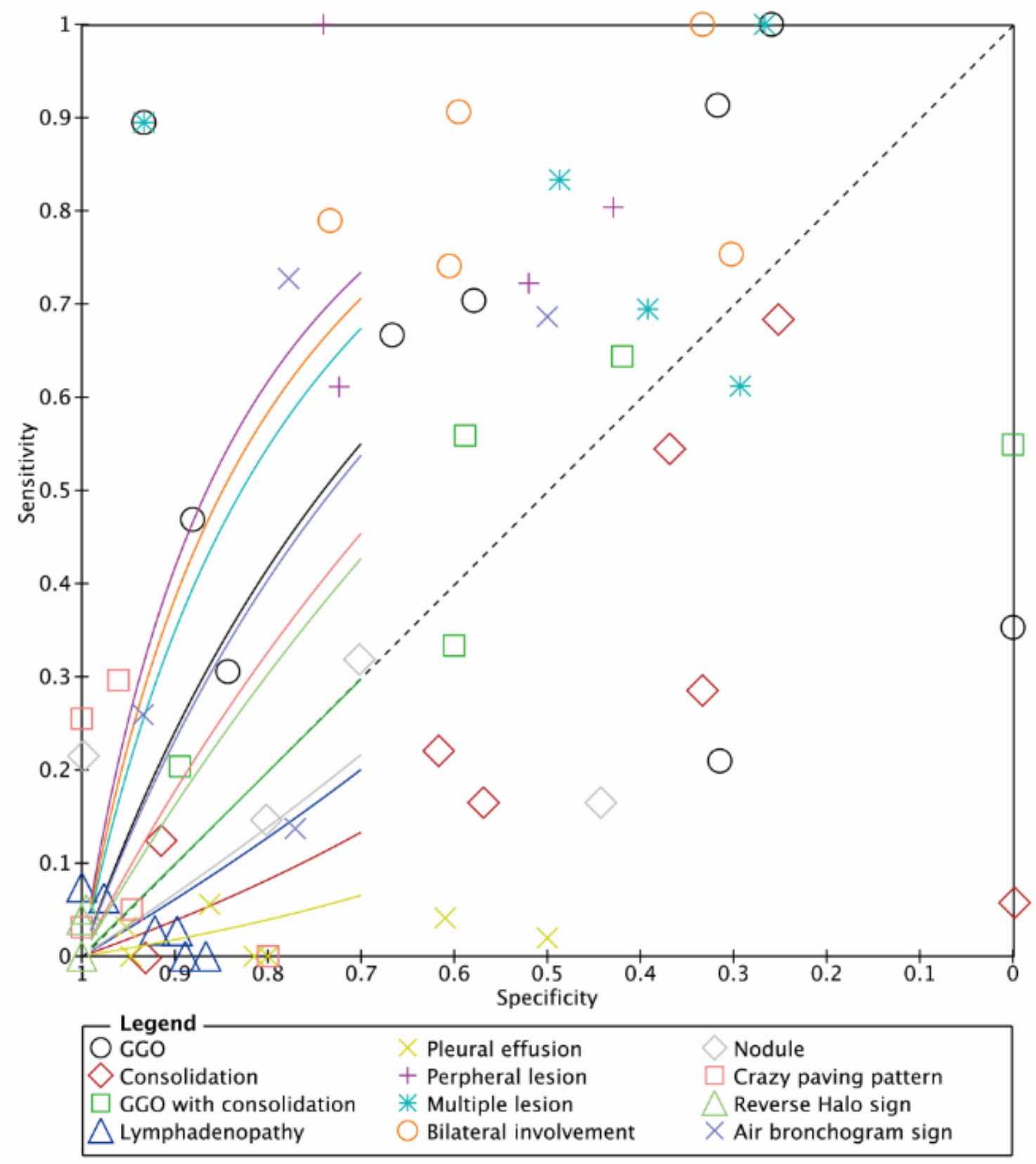

Figure 5

Symmetric summary receiver operating characteristic (symmetric SROC) of chest CT imaging features for diagnosing COVID-19

\section{Supplementary Files}

This is a list of supplementary files associated with this preprint. Click to download.

- sFig.3PublicationBias.tif

- sTable1.Thedetailedresearchstrategy.docx

- sFig.1Flowchart.tif

- sFig.2Methodologicalqualitysummary2.tif 